\title{
Defining the rehabilitation adherence curve and adherence phases of stroke patients: an observational study
}

This article was published in the following Dove Press journal:

Patient Preference and Adherence

21 August 2017

Number of times this article has been viewed

\section{Meiqi Yao \\ Jinhua Chen \\ Jiyong Jing \\ Han Sheng \\ Xing Tan \\ Jingfen Jin}

Nursing Department, The Second Affiliated Hospital, School of Medicine, Zhejiang University, Hangzhou,

Zhejiang Province, People's Republic of China
Correspondence: Meiqi Yao; Jingen Jin Nursing Department, The Second Affiliated Hospital, School of Medicine, Zhejiang University, No 88, Jiefang Road, Hangzhou 310000, Zhejiang Province, People's Republic of China

Tel $+8657 \mid 87783887$

Fax +86 57I 87783568

Email zrhlyj@zju.edu.cn; zrjzkhl@zju.edu.cn

Background: Patient adherence is a crucial determinant of rehabilitation in the long term after stroke. However, adherence is inconstant and fluctuates along a time course, and the underlying regular pattern of adherence variation remains to be clarified.

Objective: We aimed to describe the longitudinal pattern of adherence to rehabilitation exercises in stroke patients and to determine different adherence phases based on formulated rehabilitation adherence curve.

Patients and methods: Rehabilitation adherence levels were prospectively collected using the Questionnaire of Exercise Adherence (EAQ) among patients diagnosed with first-onset stroke since the second week of stroke onset, with a follow-up of 24 weeks. SPSS19.0 was used to formulate a fitting curve based on a scatter diagram. Possible causal factors for the different adherence phases are also discussed from the psychological, socioeconomic, and behavioral aspects.

Results: A total of 98 patients were included in this study. General adherence of the included subjects was classified as low to medium during follow-up. The adherence fitting curve was an "S" curve, with the fitting function $y=0.005 x^{3}-0.211 x^{2}+1.963 x+52.345$. Three phases, namely, rapid increase phase, slow decrease phase, and stable phase, were identified based on the adherence curve, and relevant theories are explored.

Conclusion: Rehabilitation adherence of stroke patients is a dynamic behavioral process that continuously changes along a time course, with a regular pattern of an "S" curve and includes a rapid increase phase, a slow decrease phase, and a stable phase.

Keywords: cerebrovascular disease, rehabilitation pattern, early rehabilitation

\section{Introduction}

Stroke is a global health threat with high incidence, mortality, and disability rate. ${ }^{1,2}$ An epidemiology study reveals that $>70 \%$ of stroke survivors suffer from functional disabilities of different levels, among which severe disability accounts for $40 \%,{ }^{3}$ resulting in serious damage to the quality of life as well as severe socioeconomic burden. ${ }^{4}$ According to the Global Atlas on Cardiovascular Disease Prevention and Control of 2011, ${ }^{5}$ lack of exercise is a major risk factor for stroke recurrence and delayed recovery. Studies suggest that functional exercise is the main strategy to help patients regain the ability to handle daily activities and is the first choice of therapy for stroke rehabilitation. ${ }^{6,7}$ Although the benefits of long-term exercise have been proven, patient adherence to rehabilitation exercise is not ideal. ${ }^{8}$ According to the WHO, patient adherence is defined as “the extent to which a person's behavior - taking medication, following a diet, and/or executing lifestyle changes - corresponds with agreed 
recommendations from a health care provider". ${ }^{9}$ However, in the management of chronic diseases such as heart failure, patient adherence to medical advice is not ideal and decreases over time. ${ }^{10,11}$ On the other hand, clinical observation in stroke rehabilitation suggests that patient adherence to medications and exercise is relatively satisfactory and that limb function recovery is relatively remarkable during hospitalization in the initial stage after stroke onset. ${ }^{12-14}$ Yet after discharge, adherence to rehabilitation therapy declines in most patients, leading to poor outcomes in terms of limb rehabilitation. Based on this finding, the hypothesis was proposed that adherence to functional exercise of the stroke patient is a continuously changing dynamic behavioral process. In order to explore the regular pattern of adherence to rehabilitation exercise by stroke patients, 98 patients were prospectively included in this study with a follow-up of 24 weeks. As a result, patient adherence fluctuated from a low level to a high level and then decreased to a low level again and remained stable at the low level. The fitting curve was an "S" curve comprising three phases: rapid increase phase, slow decrease phase, and stable phase.

\section{Patients and methods}

\section{Patients}

Stroke patients admitted to the Second Affiliated Hospital of Zhejiang University School of Medicine between August 2015 and March 2016 were enrolled in this study. The inclusion criteria were as follows: 1) first stroke diagnosed by three neurologists and confirmed by computed tomography (CT) and/or magnetic resonance imaging (MRI); 2) within 2 weeks after symptom onset; 3 ) hemiplegic paralysis with lateral muscle strength of 3/5 or lower; 4) aged 18-90 years old; and 5) written informed consent was provided. Exclusion criteria were as follows: 1) patients not equipped with telephone or other communication equipment; 2) concurrent psychiatric disorders; 3 ) severe comorbidities that are disabling, restricting their daily activities, or shortening life expectancy, including malignancy as well as heart, liver, kidney, lung or other major organ dysfunction; and 4) pregnancy and lactation. The study was approved by the Ethical Committee of the Second Affiliated Hospital of Zhejiang University School of Medicine.

\section{Methods}

\section{Measurement tools}

1. Baseline characteristics recording form: including name, sex, education, marital status, career, income, health care payment methods, living arrangement, registered permanent residence, family history, diagnosis, carer, comorbidities, primary presenting symptoms, whether receiving rehabilitation guidance, and so on.

2. Functional rehabilitation exercise adherence rating scale: Questionnaire of Exercise Adherence (EAQ) ${ }^{12}$ (Supplementary material) was used in this study. EAQ was designed by Lin et $\mathrm{al}^{12}$ in 2013 . The content validity index of the scale is 0.95 , Cronbach's $\alpha$ value is 0.923 , and the intraclass correlation coefficients range from 0.788 to $0.850 .{ }^{12}$ EAQ meets the requirements of the current study and has therefore been adopted in this research with the agreement of its original authors.

The EAQ consists of 14 items in three dimensions: adherence to rehabilitation exercise (eight items), effective supervision (three items), and advice seeking (three items). Each item has a score of one to four: the score of one ("never") indicates that the patient was unable to complete the corresponding guidance; two ("sometimes") indicates that the patient completes the guidance in $<50 \%$ of situations; three ("often") means $>50 \%$ but $<100 \%$; and four ("always") means always being able to complete the guidance or training requirements.

According to the instruction of the EAQ,

$$
\begin{aligned}
& \text { Adherence index } \\
& =\frac{\text { Actual adherence scores }}{\text { Expected maximum adherence scores }} \times 100
\end{aligned}
$$

In this formula, the expected maximum adherence score is the full score for each item, which equals four points $\times 14$ items $=56$ points. The actual adherence scores were recorded by trained investigators via telephonic or face-to-face interviews. Higher scores represent higher levels of adherence: scores $>75.0,50.0-75.0$, and $<50.0$ indicate high, medium, and low levels of adherence, respectively.

\section{Sample size determination}

According to the sample size estimation method proposed by Gorsuch, ${ }^{13}$ the minimum sample size is five subjects per variable. Since there are 14 independent variables in the EAQ, and considering a loss rate of $15 \%$ for follow-up, the calculated minimum sample size $\mathrm{N}=(14 \times 5) \times(1+15 \%)=80.5$. In order to minimize errors, an actual sample size of 98 was determined.

\section{Data collection}

\section{Baseline characteristics}

With the approval of the ethical committee, patients admitted to the Second Affiliated Hospital of Zhejiang University School of Medicine were screened according to the inclusion 
and exclusion criteria, and written informed consent was obtained from each subject. Collection of baseline characteristics was completed before discharge.

Functional rehabilitation exercise adherence

Trained investigators scored each patient by the EAQ every

2 weeks, from the second week to the 24th week continuously after stroke onset. The investigators were not blinded to the study hypothesis.

\section{Quality control}

All the investigators were trained to understand the EAQ and the instruction guidelines of the scale by the principal investigator before the beginning of the study. Patients were evaluated face-to-face during hospitalization and were reviewed by telephone after discharge. All the data were checked by two independent investigators and were fed into computers on the same day of data collection.

\section{Statistics}

Baseline characteristics were described by percentage. The central tendency of the EAQ scores was presented as mean value, and variation was described as range and $\mathrm{SD}$. A scatter diagram was drawn with time on the $x$-axis and adherence rate on the $y$-axis, based on which a fitting curve was formulated by nonlinear regression. All the data were processed by IBM SPSS Statistics Version 19.0 (IBM Corporation, Armonk, NY, USA).

\section{Results}

\section{Patient profile and baseline characteristics}

A total of 98 patients were enrolled in this study, among which one case died, seven cases withdrew during the study, and three cases were lost to follow-up. Thus, 87 patients completed the study. Among the patients, there were 61 males (70.11\%) and 26 females (29.88\%); the mean age was $65.67 \pm 10.20$ (range: $31-88$ ) years; 62 patients $(71.26 \%$ ) presented with ischemic stroke and 25 patients $(28.73 \%)$ presented with hemorrhagic stroke. All of the patients were discharged to their respective homes.

\section{General adherence levels}

During the 24-week follow-up, functional exercise adherence rate remained within medium-to-low levels: adherence rate reached the highest levels at the sixth week after stroke onset, which was $59.90 \%$, belonging to the medium level; from the 14th week, patient adherence rate was lower than the initial levels during hospitalization; the adherence rate

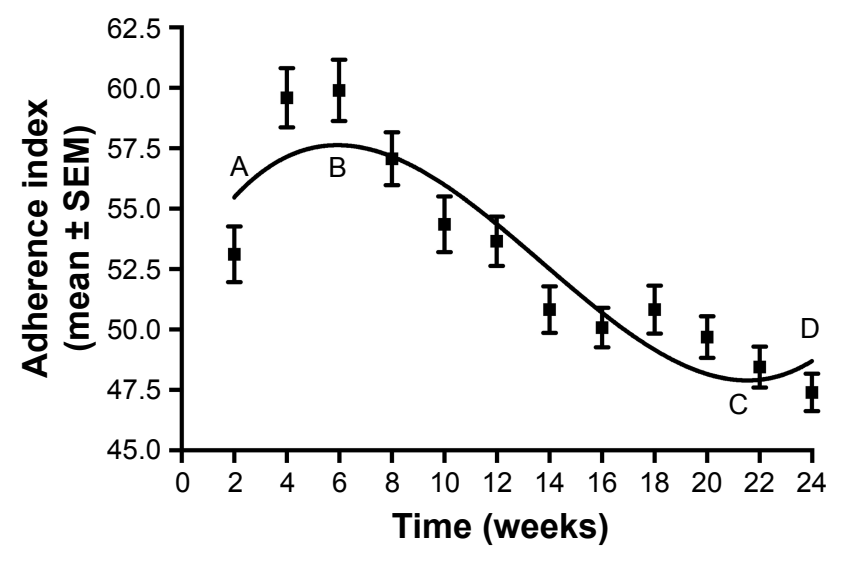

Figure I Observed adherence rate over time and the adherence fitting curve. Abbreviation: SEM, standard error of the mean.

remained $<50 \%$ after the 16 th week, belonging to the low level (Figure 1).

\section{Central tendency and variation}

During the observation period from the second to the 24th week after stroke, patient adherence index was normally distributed and fluctuated between 32.14 and 85.71 ( mean $=52.90$, range $=53.57, \mathrm{SD}=10.30$ ).

\section{Adherence curve}

The adherence curve was fitted using a one-variable cubic function. The fitting function was $y=0.005 x^{3}-0.211 x^{2}+$ $1.963 x+52.345$. As Figure 1 shows, adherence was a dynamically fluctuating process, presenting as an "S" curve.

\section{Adherence phases}

The adherence curve was composed of three phases, namely, the rapid increase phase ( $\mathrm{AB}$ in the figure), the slow decrease phase ( $\mathrm{BC}$ in the figure), and the stable phase (CD), which marked the three phases of the adherence fluctuation process.

\section{Discussion}

\section{Formation of adherence curve and} adherence phases

\section{Rapid increase phase $(A B)$}

This phase included the period between the first and the sixth weeks after stroke. During this period, patients were mostly hospitalized in the intensive care unit, emergency department, neurology department, or rehabilitation department. After 2 weeks of acute treatments in hospital, patients had a strong will to survive and recover, as well as strong confidence in rehabilitation exercise. Meanwhile, this period was also the best time for functional recovery, ${ }^{6}$ and rehabilitation therefore exerted remarkable effects. In addition, during 
this period, patients received comprehensive guidance and supervision of doctors and nurses, so their adherence levels increased rapidly.

\section{Slow decrease phase $(B C)$}

During the sixth to the 21 st week, most patients were discharged from the hospital and returned to their homes. However, most families did not have rehabilitation equipment and instruments, and most family members were unable to provide professional, and sufficient, guidance and supervision as with the doctors and nurses. Consequently, the adherence levels to rehabilitation exercises of patients changed. On the other hand, subjective factors like inertia and loss of interest also substantially impaired rehabilitation adherence. Since patients came from different families, and because the time and extent of the herein-mentioned problems were different, the decrease of adherence was relatively slow, and took 12 weeks, approximately.

\section{Stable phase (CD)}

After a rapid increase and a slow decrease, no drastic fluctuation occurred during the 21 st to the 24 th weeks. In this stage of stroke recovery, neurological function restoration was difficult and the effects of rehabilitation were not as obvious as before. Moreover, at this time, patients had become accustomed to their lifestyle after stroke. Without intervention, their adherence may continue to remain at this level and new health problems may occur.

\section{Theoretical explanations for the phases of adherence}

\section{Psychological explanations}

Willpower

Willpower, or volition, is the psychological process by which individuals ascertain their objectives, control and regulate their behavior related to the objectives, and overcome difficulties. ${ }^{14}$ Willpower is the dominant essence that motivates and regulates human behavior, including rehabilitation exercise. However, willpower is a dynamic psychological process that fluctuates periodically following a "willpower curve". ${ }^{15}$ Rehabilitation exercise is a long-term tedious process and requires willpower to maintain the adherence. Due to the existence of the willpower curve, the existence of the adherence curve is acceptable.

\section{Halfway effect}

In the behavioral process toward a specific target, an extremely fragile point lies at approximately the halfway mark, and termination of the behavioral process often happens around this point. In psychological studies, this phenomenon is defined as the "halfway effect". ${ }^{16}$ Two main reasons are attributed to the halfway effect: the target and the willpower of the individual. If the target is irrational, or if the patient has weak willpower, the halfway effect is likely to occur. Due to the acute feature of stroke, its sudden onset changes an individual from being healthy to becoming disabled abruptly. Therefore, after the onset, the patients hope to recover quickly and become healthy again. As a result, during the first 8 weeks, a rapid increase occurs in the adherence curve. Unfortunately, rehabilitation is a long-term process. After a certain period, the patients are likely to find that they fail to reach their ideal goal and may therefore give up their rehabilitation. During this period, the adherence rate declines gradually and forms the slow decrease phase.

\section{Effects of success or failure}

Psychological studies find that previous success contributes to subsequent success, whereas failure contributes to subsequent failure, which is defined as "effects of success or failure". ${ }^{20}$ In stroke rehabilitation, the effects during the early stage are more obvious than at the later stages. Therefore, during the early stage, patients may be more confident and active in rehabilitation, which is the "effect of success" On the other hand, with the gradual declining effects of rehabilitation, they may become disappointed due to the "effects of failure". Hence, adherence levels decrease gradually and remain at a low level in the delayed phase.

\section{Sociology explanations}

Traditional culture of family and filial piety

Filial piety is one of the key virtues in Chinese culture, which mainly refers to respect and obedience to parents and elders. After the onset, most patients are taken care of by their children at home instead of going to nursing homes. Therefore, family is the main source of social support and rehabilitative care for patients. However, family members cannot provide sufficient health care forever due to various reasons. Adherence correlates with social support, ${ }^{17}$ which partially explains the formation of the adherence curve. In the initial stage after stroke, adherence increases gradually with support from family, followed by slowly descending filial piety and, correspondingly, adherence.

\section{Empty nest phenomenon}

With the progressing tendency of population aging, urbanization, and decrease in family size, the group of empty nest 
elderly is substantially growing in China. Currently, empty nesters account for $52.2 \%$ and $46.5 \%$ of rural and urban elderly, respectively; this figure has increased from $27.9 \%$ in 2007 and is expected to reach $90 \%$ by $2030 .{ }^{18,19}$ In empty nest patients, rehabilitative support relies mainly on doctors and nurses during hospitalization. Once discharged, their quality of life and rehabilitation training will be impaired. Therefore, the empty nest phenomenon in current society also contributes to the existence of the adherence curve and different phases of adherence.

\section{Behavioral theories}

Positive reinforcement and negative reinforcement

In behavioral psychology, reinforcement is described as the consequence of a previous behavior that can influence subsequent behavior. When the previous behavior leads to a positive effect, the specific behavior is strengthened and consolidated gradually, which is referred to as positive reinforcement. However, the reverse is also true and is defined as negative reinforcement. ${ }^{20}$

The effect of early rehabilitation training on neurological function recovery is obvious during the first to the eighth weeks after a stroke, when positive reinforcement is dominant. As a result, patient adherence rises during this phase. In the latter stage of recovery, functional improvement is slow and rehabilitation training seems ineffective, so negative reinforcement plays a major role and consequently forms the slow decrease phase (9-20 weeks) and the stable phase (21-24 weeks).

\section{Behavior control}

Human behavior requires appropriate control and regulation, which demands both subjective willingness and objective guidance. In the field of stroke rehabilitation, adherence behavior also needs appropriate control and regulation. During hospitalization, patients receive guidance from doctors and nurses, which guarantees the increment of adherence in the early stage. However, due to the restricted health care resources in China, patients are discharged in a few weeks and behavior control mainly relies on the subjective willingness of individuals. In the absence of proper guidance, behavior control - and hence adherence - may decline.

\section{Limitations}

There were several limitations in this study. Firstly, it was a single-center study with a small sample size and 6 months of follow-up. Although the sample size was determined by scientific methods, ${ }^{13}$ our findings require further confirmation in larger and multicenter studies with longer follow-up periods. Secondly, we exclusively focused on stroke patients, which limited the application of our findings to the rehabilitation of patients with other diseases. Finally, the study faced a methodological dilemma: the investigators interviewed the patients every other week, which was a reminder for the patients to persist with their rehabilitation exercise. With the reminding effect, the adherence index collected in the study was better than that in real clinical practice. However, a clear decline still occurred in our observation period, indicating that it was an appropriate timing for clinical intervention for rehabilitation to maintain the adherence at a satisfactory level.

\section{Conclusion}

Adherence to rehabilitation training of stroke patients is a dynamic process, which consists of three phases: rapid increase phase, slow decrease phase, and stable phase. This phenomenon may result from psychological, sociological, and praxeological reasons. The recognition of the adherence curve and the various phases has improved the concept of rehabilitation adherence, expanded the understanding of rehabilitation training, and provided evidence and reference for rehabilitative care in clinical practice.

\section{Acknowledgments}

This study was financially supported by the Health Science and Technology Special Fund of Zhejiang Province (2015KYA106). We thank Ms Lin from the Zhengzhou University for permitting the use of the Questionnaire of Exercise Adherence in the current study. There were no financial benefits to the authors. This research work has not been previously presented anywhere in manuscript or abstract form.

\section{Disclosure}

The authors report no conflicts of interest in this work.

\section{References}

1. Bennett DA, Krishnamurthi RV, Barker-Collo S, et al; Global Burden of Diseases, Injuries, and Risk Factors 2010 Study Stroke Expert Group. The global burden of ischemic stroke: findings of the GBD 2010 study. Glob Heart. 2014;9(1):107-112.

2. Toyoda K, Koga M, Hayakawa M, Yamagami H. Acute reperfusion therapy and stroke care in Asia after successful endovascular trials. Stroke. 2015;46(6):1474-1481.

3. Liu M, Wu B, Wang WZ, Lee LM, Zhang SH, Kong LZ. Stroke in China: epidemiology, prevention, and management strategies. Lancet Neurol. 2007;6(5):456-464

4. Krishnamurthi RV, Feigin VL, Forouzanfar MH, et al; Global Burden of Diseases, Injuries, Risk Factors Study 2010 (GBD 2010); GBD Stroke Experts Group. Global and regional burden of first-ever ischaemic and haemorrhagic stroke during 1990-2010: findings from the global burden of disease study 2010. Lancet Glob Health. 2013;1(5):e259-e281. 
5. Mendis S, Puska P, Norrving B, Mendis S, Puska P, Norrving B. Global Atlas on Cardiovascular Disease Prevention and Control. Geneva: World Health Organization; 2012.

6. Salter K, Jutai J, Hartley M, et al. Impact of early vs delayed admission to rehabilitation on functional outcomes in persons with stroke. $J$ Rehabil Med. 2006;38(2):113-117.

7. Messaggi-Sartor M, Guillen-Sola A, Depolo M, et al. Inspiratory and expiratory muscle training in subacute stroke: a randomized clinical trial. Neurology. 2015;85(7):564-572.

8. Prout EC, Mansfield A, McIlroy WE, Brooks D. Patients' perspectives on aerobic exercise early after stroke. Disabil Rehabil. 2016;39(7): 684-690.

9. De Geest S, Sabate E. Adherence to long-term therapies: evidence for action. Eur J Cardiovasc Nurs. 2003;2(4):323.

10. Artinian NT, Magnan M, Sloan M, Lange MP. Self-care behaviors among patients with heart failure. Heart Lung. 2002;31(3):161-172.

11. Hu X, Hu X, Su Y, Qu M, Dolansky MA. The changes and factors associated with post-discharge self-care behaviors among Chinese patients with heart failure. Patient Prefer Adherence. 2015;9:1593-1601.

12. Lin B, Zhang Z, Sun Y, Mei Y, Xie J, Zhang Y. [A new scale evaluating functional exercise compliance for stroke patients in community: design, reliability and validity tests]. Chin J Rehabil Med. 2013;28(6): 574-578. Chinese.
13. Gorsuch R. Factor Analysis. 2nd ed. Hillsdale: Lawrence Erlbaum Associates; 1983.

14. Henderson S. The neglect of volition. Br J Psychiatry. 2005;186: 273-274.

15. Baumeister RF, Tierney J. Willpower: Rediscovering the Greatest Human Strength. New York: Penguin Press; 2011.

16. Hu J. ["Half-way effect" and perfect inspiration]. Mod Ind Econ Info. 2011;11:67-67. Chinese.

17. Lemstra M, Rogers MR. The importance of community consultation and social support in adhering to an obesity reduction program: results from the Healthy Weights Initiative. Patient Preference Adherence. 2015;2015(9):1473-1480

18. Sun X, Lucas H, Meng Q, Zhang Y. Associations between living arrangements and health-related quality of life of urban elderly people: a study from China. Qual Life Res. 2011;20(3):359-369.

19. Cheng P, Jin Y, Sun H, et al. Disparities in prevalence and risk indicators of loneliness between rural empty nest and non-empty nest older adults in Chizhou, China. Geriatr Gerontol Int. 2015;15(3):356-364.

20. Schultz W. Neuronal reward and decision signals: from theories to data. Physiol Rev. 2015;95(3):853-951. 


\section{Supplementary material Questionnaire of Exercise Adherence (EAQ)}

Questionnaire instructions: please tick in the box if you agree to the terms. The term "never" means you are unable to complete the corresponding guidance; "sometimes" means that you adhere to the guidance on $<50 \%$ occasions; "often" means you adhere to the guidance on $>50 \%$ occasions, but have not yet reached $100 \%$; and "always" means you can complete the guidance or training requirements if there is no special situation. Thanks for your cooperation!

1. Follow the instructions given by the medical staff during admission and after discharge.

$\square$ (1) never $\square$ (2) sometimes $\square$ (3) often $\square$ (4) always

2. Follow the guidance strictly and insisting on exercising 2-3 times every day at least.

$\square$ (1) never $\square$ (2) sometimes $\square$ (3) often $\square$ (4) always

3. Strictly follow the requirement that each exercise duration be for at least $30-45$ minutes.

$\square$ (1) never $\square$ (2) sometimes $\square$ (3) often $\square$ (4) always

4. Insist on walking up and down the stairs every day (or other forms of exercise that can be done).

$\square$ (1) never $\quad \square$ (2) sometimes $\square$ (3) often $\square$ (4) always

5. Insist on practicing dressing, eating, washing face, and brushing teeth by yourself (especially practicing the function of the paralyzed side).

$\square$ (1) never $\square$ (2) sometimes $\square$ (3) often $\square$ (4) always
6. Adhere to exercise every day to the paralyzed side of the body.

$\square$ (1) never $\quad \square$ (2) sometimes $\quad \square$ (3) often $\square$ (4) always

7. Stretch the affected extremity to keep it in antispasmodic condition.

$\square$ (1) never $\square$ (2) sometimes $\square$ (3) often $\square$ (4) always

8. Care about your own functional recovery of the affected extremity and push yourself to exercise daily.

$\square$ (1) never $\square$ (2) sometimes $\square$ (3) often $\square$ (4) always

9. You take the initiative to observe and evaluate the effect of limb functional exercise.

$\square$ (1) never $\square$ (2) sometimes $\square$ (3) often $\square$ (4) always

10. You adhere to the learning of healthy exercises regularly. $\square$ (1) never $\square$ (2) sometimes $\square$ (3) often $\square$ (4) always

11. During the functional exercise, you accept the family/ friends' advice.

$\square$ (1) never $\square$ (2) sometimes $\square$ (3) often $\square$ (4) always

12. In the process of functional exercise, you like to talk with the medical staff when you encounter problems.

$\square$ (1) never $\square$ (2) sometimes $\square$ (3) often $\square$ (4) always

13. You communicate with your wardmates who are just like you.

$\square$ (1) never $\square$ (2) sometimes $\square$ (3) often $\square$ (4) always

14. When you notice that there is no positive effect of functional exercise, you're eager to seek suggestions from others to improve the effectiveness of functional exercise. $\square$ (1) never $\square$ (2) sometimes $\square$ (3) often $\square$ (4) always

Patient Preference and Adherence

Dovepress

\section{Publish your work in this journal}

Patient Preference and Adherence is an international, peer-reviewed, open access journal that focuses on the growing importance of patient preference and adherence throughout the therapeutic continuum. Patient satisfaction, acceptability, quality of life, compliance, persistence and their role in developing new therapeutic modalities and compounds to optimize clinical outcomes for existing disease states are major areas of interest for the journal. This journal has been accepted for indexing on PubMed Central. The manuscript management system is completely online and includes a very quick and fair peer-review system, which is all easy to use. Visit http://www. dovepress.com/testimonials.php to read real quotes from published authors. 